\title{
Runway performance as a function of the schedule and magnitude of water reward
}

\author{
PATRICK E. CAMPBELL, THOMAS A. HINSON, and BRIAN M. KRUGER \\ Wright State University, Dayton, Ohio 45431
}

\begin{abstract}
Sixty rats were trained in a runway for $\mathbf{4 2}$ days of acquisition and 15 days of extinction at the rate of one trial a day. Half of the animals received a $50 \%$ partial reinforcement (PR) schedule and half received a $100 \%$ reinforcement schedule (CRF). For one-third of the rats within each schedule the magnitude of water reward was $.1, .4$, or $.8 \mathrm{ml}$. Persistence during extinction was an increasing function of reward magnitude for the PR animals, but a decreasing function of reward magnitude for the CRF animals. A partial reinforcement extinction effect was observed with the medium and large reward but not with the smallest reward. These data are consistent with the literature dealing with food reward.
\end{abstract}

In a recent article, MacDonald and de Toledo (1974) observed greater persistence following partial reinforcement (PR) than after continuous reinforcement (CRF) when the reward was food but not when the reward was water. Their failure to obtain the familiar partial reinforcement extinction effect (PREE) with water led MacDonald and de Toledo to speculate concerning a possible biological constraint on the development of behavioral persistence. Extrapolating from a suggestion by Petrinovich and Bolles (1954) that rats, in their natural environment, continually explore for food but enjoy a relatively fixed water supply, MacDonald and de Toledo suggested that behavioral persistence might develop more easily with food reward than with water reward. In their experiments, involving both a freeoperant barpress task and a discrete-trial runway response, they observed the PREE with food (a single $45-\mathrm{mg}$ Noyes pellet) but not with water (approximately $.1 \mathrm{ml}$ ). These results, of course, are consistent with the idea of a biological constraint on the development of persistence.

Nevertheless, behavioral persistence, and specifically the PREE, are sensitive to a wide variety of experimental parameters. At least one of these variables was not at an optimum level in the MacDonald and de Toledo studies. It is well documented that the extent of the PREE increases with the magnitude of food reward (cf. Robbins, 1971). Two complementary processes are responsible for this relationship. Persistence is clearly a decreasing function of the magnitude of food reward in CRF schedules and generally an increasing function of the magnitude of food reward in PR schedules. It is possible that MacDonald and de Toledo (1974) failed to

Requests for reprints should be sent to Patrick E. Campbell, Department of Psychology, Wright State University, Dayton, Ohio 45431. observe a PREE with water because their use of $.1 \mathrm{ml}$ amounted to a small magnitude of reward. In their discussion, MacDonald and de Toledo considered this possibility but rejected it, since they believed that their study involved a large magnitude of reward. Nevertheless, several considerations suggest that a $.1-\mathrm{ml}$ water reward is a small reward, at least in the alleyway situation. By the end of 80 trials of acquisition, their rats were running at speeds of approximately $20 \mathrm{~cm} / \mathrm{sec}$. In the typical runway situation, speeds of $100 \mathrm{~cm} / \mathrm{sec}$ or faster are commonly reported even with small food rewards. In addition, their water-trained animals did not differ in acquisition running speeds from the animals that were rewarded with only one $45-\mathrm{mg}$ Noyes pellet. Of course, in the reward magnitude literature a single 45-mg pellet is considered a small reward.

The purpose of the present study was to further investigate the occurrence or nonoccurrence of the PREE with schedules involving water as the reward. A straight-alley runway was used and the reward magnitude was varied across three levels between .1 and $.8 \mathrm{ml}$. Only one trial was given each day to eliminate any problem with differential satiation.

\section{METHOD}

Sixty naive female rats of the Sprague-Dawley strain were purchased from Blue-Spruce Farms, Altamont, New York. They were housed in individual cages in a room with a 10-h-off/ 14-h-on light cycle. All sessions were conducted during dark phases of the cycle.

The $160 \times 13 \times 9 \mathrm{~cm}$ straight runway and $37 \times 13 \times 9 \mathrm{~cm}$ goalbox were painted flat medium gray throughout and covered with clear plastic lids. The 4-cm-long goal area of the goalbox contained a 15-mm-diam, 8-mm-deep water cup and was separated from the $33-\mathrm{cm}-$ long confinement area by a guillotine door. The confinement area was separated from the runway by a second guillotine door. A system of photoelectric relays and .01-sec clocks measured start, run, and goal times for consecutive 25-, 100-, and $25-\mathrm{cm}$ distances, respectively. The first infrared beam was located $25 \mathrm{~cm}$ from the start end of the 
alley and the last was $20 \mathrm{~cm}$ inside the confinement area. Two $25-\mathrm{W}$ red lamps located $1 \mathrm{~m}$ above the goal area provided the illumination, and a 75-dB (SPL) white masking noise was present during sessions.

Upon arrival, all rats were given ad-lib access to food and water. After $48 \mathrm{~h}$ all were placed on a $23-1 / 2-\mathrm{h}$ water-deprivation schedule for 3 days prior to pretraining and for the first 3 days of pretraining. Thereafter, all subjects were maintained on a 23-2/3-h deprivation schedule. On pretraining and training days the 30 - or $20-\mathrm{min}$ free access to water was given $30 \mathrm{~min}$ after the session. Subject weights stabilized by the end of pretraining.

All animals were handled individually for $2 \mathrm{~min}$ each day prior to pretraining. On Days $1-6$ of pretraining, groups of three were placed in the starting area and allowed to explore the runway and baited goalbox. On Days 1-3 they were given $5 \mathrm{~min}$, while on Days 4-6 they were given 2 min or until they individually drank. On Days 7-8 individuals were given 2 min to enter the goalbox. Once in the goalbox, they were confined there for $1 \mathrm{~min}$ or until they drank. If an animal failed to enter the goalbox within $2 \mathrm{~min}$, it was placed there manually. On Days 9-10 individuals were given one confinement in the goalbox without runway exploration. On Day 11 individuals were given up to $1 \mathrm{~min}$ to explore the runway before confinement. During individual pretraining (Days 7-11) the goalbox was baited with the amount of water used throughout training for reinforced trials. All subjects learned to drink from the water cup during pretraining.

During the acquisition phase, one trial a day was given for 42 days. Rats in the CRF groups received $.1, .4$, or $.8 \mathrm{ml}$ of water reinforcement on every trial. Rats in the three PR groups were reinforced with $.1, .4$, or $.8 \mathrm{ml}$ of water on $50 \%$ of the trials. The sequence of reinforced and nonreinforced trials was determined randomly, with the restriction that no more than three consecutive reinforced or nonreinforced trials could occur. In extinction 15 nonreinforced trials were administered at one trial per day beginning the day after the last acquisition trial.

Trials were begun by placing the rat in the starting area prior to the first infrared beam. On all trials, goalbox confinement began and the door to the goal opened when the rat interrupted the beam in the goalbox. Rats not traversing a section of the runway within $30 \mathrm{sec}$ were placed in the goalbox and a time of $30 \mathrm{sec}$ was recorded for each section not traversed. On nonreinforced trials subjects were confined for $1 \mathrm{~min}$ in the goalbox. On reinforced trials the rats were confined until the water was consumed.

The rats were run in squads with one rat from each of the six experimental groups. The squads were run in the same order each day but the running order within squads changed daily.

\section{RESULTS}

The times for each segment and the total times were converted to speeds prior to analysis. Since the results for the total speed measures were quite representative of the three segment measures, only the total results are presented.

The acquisition response speeds for each of the reward magnitude by schedule combinations were averaged for six-trial blocks and are presented in Figure 1. An inspection of the figure suggests approximately equal performance for the two schedules when a small reward was used, faster speeds for the CRF animals with medium reward, and faster speeds for the PR animals with large reward. In addition, running speeds tended to increase for both schedules as the magnitude

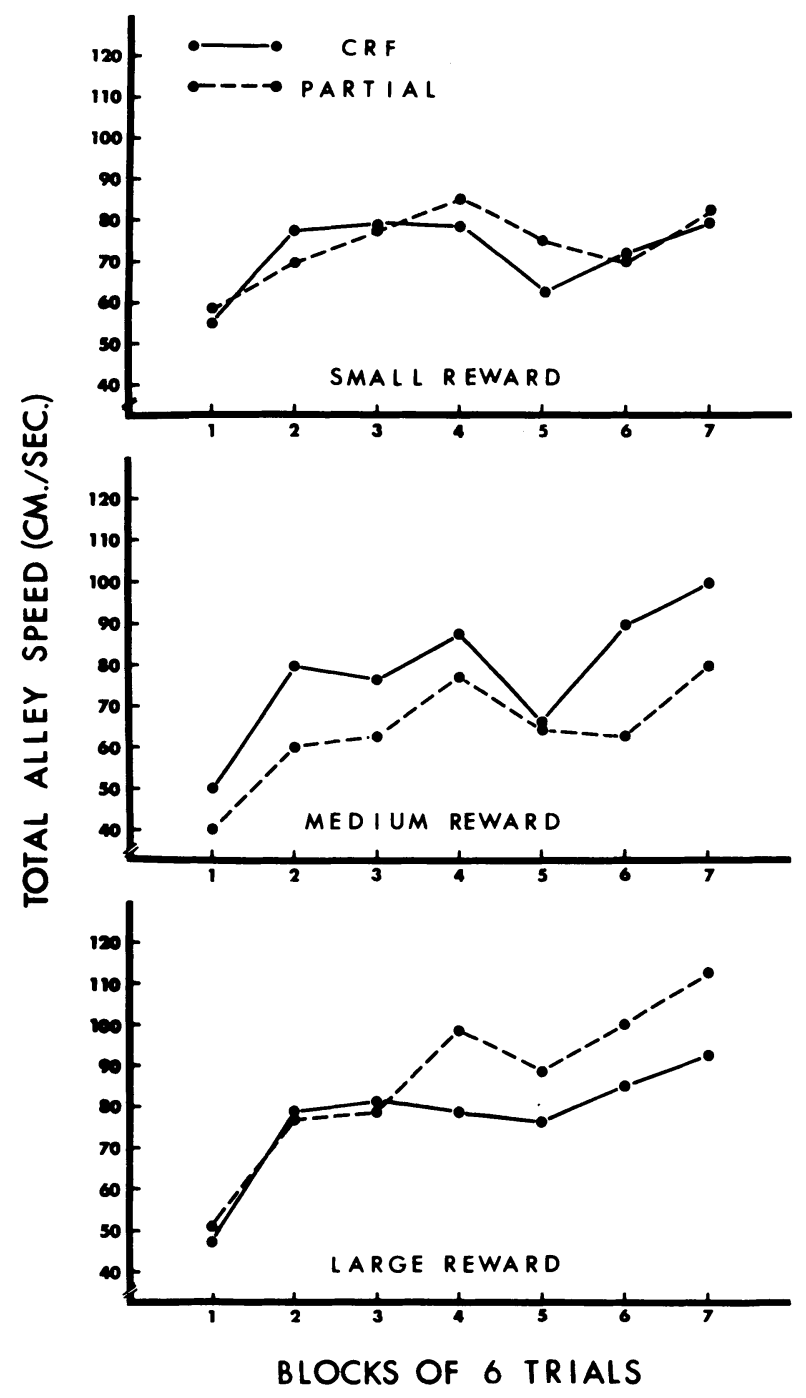

Figure 1. Mean total alley speeds for each schedule by reward magnitude combination during acquisition.

of reward increased. An analysis of variance involving the last 12 trials showed a significant main effect for the magnitude variable $(\mathrm{F}=4.06, \mathrm{df}=2 / 54, \mathrm{p}<.05)$ and a significant interaction of Schedule by Magnitude of Reward $(F=3.85, \mathrm{df}=2 / 54, \mathrm{p}<.05)$.

Since considerable differences in response speeds were present at the end of the acquisition phase, the extinction speed data were converted to a rate measure, following the suggestions outlined in Anderson (1963, Equation 4). The performance of each animal during the last block of acquisition training served as an estimate of acquisition response levels, and the maximum response time allowed during extinction was used as the extinction estimate.

The extinction rate scores were averaged for threetrial blocks and are shown in Figure 2. It is apparent that a PREE did not occur when a small reward was 

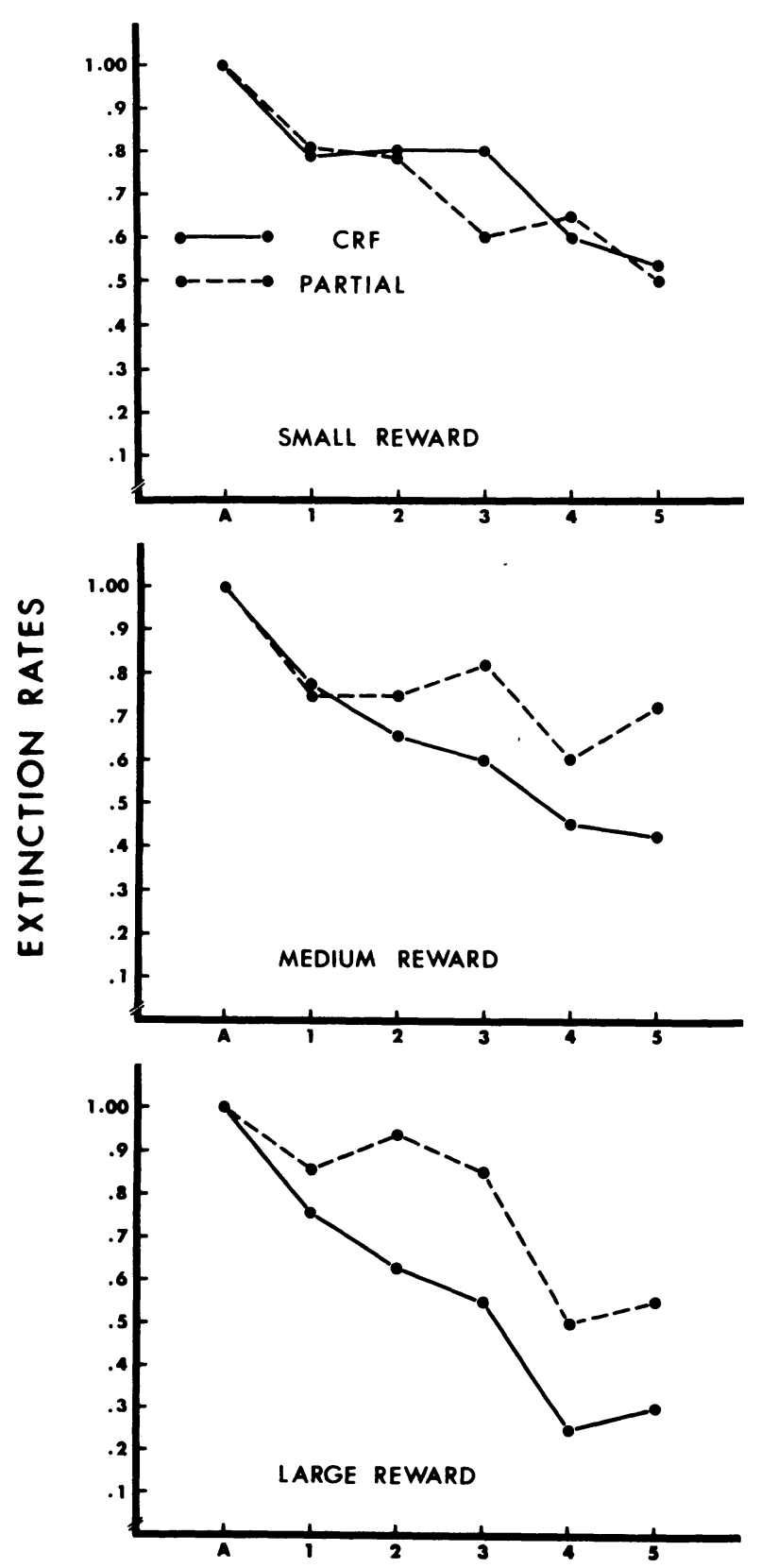

BLOCKS OF 3 TRIALS

Figure 2. Mean total alley extinction rate scores for each schedule by reward magnitude combination.

involved, but the PR animals showed slower rates of extinction than the CRF animals with both of the larger rewards. An analysis of variance revealed a significant main effect for schedule $(F=7.45, \mathrm{df}=1 / 54, \mathrm{p}<.05)$, as well as an interaction of Schedule by Magnitude of Reward $(\mathrm{F}=3.52, \mathrm{df}=2 / 54, \mathrm{p}<.05)$. Tukey HSD contrasts confirmed the occurrence of a PREE in both of the larger reward conditions $(\mathrm{p}<.05)$.

\section{DISCUSSION}

The results of the present study suggest that the extent of the PREE is an increasing function of the magnitude of water reward. This same relationship has been repeatedly observed with food reward (Gonzalez, Roberts, \& Bitterman, 1966; Hulse, 1958; Padilla, 1967; Roberts, 1969; Wagner, 1961). The present results also support MacDonald and de Toledo (1974) in not observing a PREE with a $.1-\mathrm{ml}$ reward, but they are inconsistent with their suggestion that a PREE would not occur with larger rewards. Clearly, a PREE can be obtained with water as the reinforcer. The present study demonstrated a PREE with a reward as small as $.4 \mathrm{ml}$ when rates of extinction were used.

The apparent reliability of the PREE with water is not directly contradictory to the MacDonald and de Toledo hypothesis concerning different persistence mechanisms for food and water. As they have suggested:"the presence or absence of PREE is less important than the difference between food and water as they affect rates of responding" (MacDonald \& de Toledo, 1974 , p. 296). Nevertheless, the different amounts of persistence generated by food and water in the MacDonald and de Toledo experiment might have resulted from their selection of reward magnitudes. The present data suggest that a $.1-\mathrm{ml}$ reward is just below the threshold for the development of a PREE with water. A similar threshold for food reward appears at approximately $45 \mathrm{mg}$ (Hulse, 1958). These close-tocritical reward magnitudes were the levels used in the MacDonald and de Toledo experiment. It is conceivable that the selection of larger reward magnitudes would reduce or eliminate the discrepancy between food and water rewards with respect to the development of behavioral persistence.

While our extinction results appear to be generally consistent with the literature on food reward, our acquisition results are not. Running speeds did generally increase with larger rewards but the difference between the CRF and PR animals was quite unlike the results typically observed with food. For example, CRF animals nearly always run faster than PR animals in the goal section with food reward. When the start and run sections are considered, the PR animals of ten run faster than the CRF animals, especially in the start section. In the present study no differences among the various alley sections were observed. Moreover, the PR animals ran faster than the CRF animals with the largest reward, CRF resulted in faster speeds than PR with medium reward, and no difference between the two schedules was observed with the smallest reward. We do not intend to offer an explanation for this apparent interaction at this time since we are skeptical of our results with the medium reward. The advantage of the CRF animals did not appear to develop over trials, suggesting a sampling error.

\section{REFERENCES}

ANderson, N. H. Comparisons of different populations: Resistance to extinction and transfer. Psychological Review, 1963, 70, 162-179.

Gonzalez, R. C., Roberts, W. A., \& Bitterman, M. E. Resistance to extinction in normal and extensively decorticated rats as a function of amount and percentage of reinforcement. American Journal of Psychology, 1966, 79, 542-550.

Hulse, S. H. Amount and percentage of reinforcement and duration of goal confinement - in conditioning and extinction. Journal of Experimental Psychology, 1958, 56, 48-57.

MacDonald, G. E., \& de Toledo, L. Partial reinforcement effects and type of reward. Learning and Motivation, 1974, 5. 288-298. 
Padilla, A. M. A few acquisition trials: Effects of magnitude and percent reward. Psychonomic Science, 1967, 9, 241-242.

Petrinovich, L., \& Bolles, R. Deprivation states and behavioral attributes. Journal of Comparative and Physiological Psychology, 1954, 47, 450-453.

Robbins, D. Partial reinforcement: A selective review of the alleyway literature since 1960 . Psychological Bulletin, 1971, 76, 415-431.

ROBERTS, W. A. Resistance to extinction following partial and continuous reinforcement with varying magnitudes of reward. Journal of Comparative and Physiological Psychology, 1969, 67, 395-400.

WAGNER, A. R. Effects of amount and percentage of reinforcement and number of acquisition trials on conditioning and extinction. Journal of Experimental Psychology, 1961, 62, 234-242.

(Received for publication March 9, 1977.) 